\title{
Distribution and Continuity of Developers' Contributions in OSS Projects: A Case Study
}

\author{
Zhongjie Wang $^{1}$, Dewayne E. Perry ${ }^{2}$, Xiaofei $\mathrm{Xu}^{1}$ \\ 1 Harbin Institute of Technology, Harbin, China 150001 \\ 2 The University of Texas at Austin, Austin, TX, USA 78712 \\ rainy@hit.edu.cn, perry@ece.utexas.edu, xiaofei@hit.edu.cn
}

\begin{abstract}
Open Source Software (OSS) is usually developed by geographically-distributed developers in a collaborative manner. Different developers exhibit different behaviors and make diversified contributions to OSS projects. Objective of this paper is to discover individualized characteristics and common patterns of how developers contribute to OSS projects. Continuity is used to delineate how a developer actively contributes to the project over time. Case studies on two OSS projects reveal some significant phenomena on the distribution of developers' contribution$s$ relative to absolute time and relative to the milestones (i.e., releases) of OSS projects. We have found that OSS developers' contributions exhibit the "temporal locality", and most of the releases of an OSS project are dominated by the contributions of a limited number of developers.
\end{abstract}

Keywords- Open Source Software; social development; developers' contributions; continuity; temporal distribution

\section{Introduction}

Different developers have different social and technical backgrounds. They joined an OSS project with different motivations [1, 2]. Consequently, the degree of their participation and the level of their contributions are diversified [3]. A set of individualized characteristics on the behaviors of OSS developers that distinguish one from others who belong to the same OSS project team do indeed exist [4].

Most of the recognized characteristics are closely related to externally visible behaviors that developers exhibit in the history of OSS projects. This paper is focused on exploring the personalized contributing styles of developers from a temporal dimension. A developer initiatively enforces to make contributions to OSS projects, especially code commits. Targeting at the contributions, a metric called "continuity" which delineates how frequently and consecutively a developer makes contributions to a project and is measured by the times, contributed Line of Code (LoC), and temporal distribution of his active behaviors, is studied. We introduce an approach of identifying and measuring the continuity of contributions from public data in OSS repositories, in which the continuity is refined into a set of fine-grained metrics based on the temporal distribution of the active behaviors of each developer, with respect to the absolute timeline (defined by calendar time) and the relative timeline (defined by a series of releases, or milestones, of an OSS project). Entropy is employed to give an overall measurement of the continuity. Case studies are conducted on two OSS projects (JUnit and Guava) hosted on GitHub. The identified characteristics are visualized, and developers are compared in terms of these characteristics to discover the difference and similarities among their behaviors.

This study tries to answer three research questions:

RQ1: How are a developer's contributions distributed on the absolute timeline?

RQ2: How are a developer's contributions distributed relative to the milestones of the OSS project?

RQ3: Are there distinct characteristics of different developers on the distribution/continuity of their contributions?

By case studies we find that, (1) Contributions of a developer often show temporal locality, and there are some long intervals that split a developer's contributions into stages; the more contributions a developer makes, the more evenly his contributions are distributed over time, with averagely shorter intervals; (2) Most of the releases of an OSS project are dominated by contributions of a limited number of developers, respectively, i.e., the active behaviors of a few developers contribute most of the changes of a release relative to its previous one. These would help OSS project coordinators get a good understanding on the working habits of developers in the project team, thus facilitate proactive and accurate project management activities such as task allocations.

The remainder is organized as follows. Candidate projects and the independent/dependent variables of the case study are presented in Section 2. Section 3 is the study approaches and results, followed by threats of validity in Section 4 Section 5 and 6 are the related work and conclusions. 


\section{Study Setup}

\subsection{Candidate OSS Projects}

Two OSS projects from GitHub, JUnit ${ }^{1}$ and Guava ${ }^{2}$, are selected for the case study. They are diversified in many perspectives. From the perspective of time to live, JUnit is much older for above 14 years and Guava is for nearly 6 years. From the perspective of scale, Guava is larger with more than 1,700 source files and above $400 \mathrm{~K} \mathrm{LoC}$, and JUnit is a medium project with 300-400 files and 40K LoC. From the perspective of team size, JUnit has a larger team with more than 130 developers, while Guava has a relatively smaller team. From the perspective of behavior intensity, Guava has the larger number of commits than JUnit, and on average, although Guava has a smaller team, its developers seem more active and commit more average LoC than JUnit.

\subsection{Variable Selection}

\subsubsection{Independent Variables (IVs)}

An OSS project has a team $T$ and a sequence of code commits $C S$. Every time a developer submits code to the project repository, does he carry out an active behavior explicitly exhibited as the expansion or modification of existing source code in two forms: addition and deletion.

A commit $c \in C S$ is defined as $c=\langle P C, F, d, t\rangle$, where $P C$ is $c$ 's parent commits on which $c$ 's code changes are based, and each commit might have 0,1 or multiple parent commits; $F$ is the files contained in $c$, and $\forall f \in F, l^{+}(f), l^{-}(f)$ are the code lines added into and deleted from $f$, respectively, compared with the corresponding files in $P C$; $d$ is the developer who authored the code changes in $c$; and $t$ is the commit time of $c$.

Commits of a project form a sequence in which they are sorted by commit time in chronological order. In terms of a developer $d_{i}$, all the commits authored by $d_{i}$ form a sub-sequence of $C S$ and is denoted by $C S_{i}$. Multiple developers alternately check out codes from repository and commit changes back, so their commit sequences are interwoven with each other. Denote $\varphi_{i}=\left|C S_{i}\right|$, and $\sum_{i=1}^{\tau} \varphi_{i}=|C S|$.

In GitHub, a set of commits could be grouped together as a release being a phase achievement of the project 3 Here we simply define a release $r_{j}$ as a set of commits (the number of commits is $\left.\left|r_{j}\right|\right)$. Suppose there are totally $\gamma$ releases in a project and they form a sequence $\left\langle r_{1}, \ldots, r_{\gamma}\right\rangle$ in terms of the release date.

A set of git commands are used to collec$\mathrm{t}$ above-mentioned variables from git repositories,

\footnotetext{
${ }^{1}$ https://github.com/junit-team/junit

${ }^{2}$ https://github.com/google/guava

${ }^{3}$ https://help.github.com/articles/creating-releases/
}

such as git rev-list, git diff, git show, git for-each-ref ''refs/tags' , etc.

\subsubsection{Dependent Variables (DVs)}

The dependent variables (DVs) are constructed in terms of two timelines: (1) The absolute timeline defined by calendar time; (2) The relative timeline defined by the date of releases;

For the former, the lifespan of a project is split into $N$ number of timeslots $\left\langle p_{1}, \ldots, p_{N}\right\rangle$. The length of a timeslot may be 1 day, 1 week, 1 month, or any length of time. The following are the DVs used under the absolute timeline:

(1) $V_{\text {times }}\left(d_{i}\right), V_{\text {loc }}\left(d_{i}\right)$ : a developer $d_{i}$ 's distribution vectors of the times and LoC of active contributing behaviors in $N$ timeslots, i.e., $V_{\text {times }}\left(d_{i}\right)=\left\langle\varphi_{i 1}, \varphi_{i 2}, \ldots, \varphi_{i N}\right\rangle, V_{l o c}\left(d_{i}\right)=$ $\left\langle l_{i 1}^{+}, l_{i 2}^{+}, \ldots, l_{i N}^{+}\right\rangle$.

We define $C S_{i k}=\left\{c_{j} \mid c_{j} \in C S_{i} \wedge t\left(c_{j}\right) \in p_{k}\right\}$ as the commits that belong to $C S_{i}$ (the commit sequence of $d_{i}$ ) and fall into the timeslot $p_{k}(1 \leq k \leq N)$. Then, $\varphi_{i k}=\left|C S_{i k}\right|$ is $d_{i}$ 's times of active behaviors occurring during $p_{k} ; l_{i k}^{+}=$ $\sum_{c_{j} \in C S_{i k}} l^{+}\left(c_{j}\right)$ is the total LoC that $d_{i}$ contributes during $p_{k}$, in which $l^{+}\left(c_{j}\right)=\sum_{f \in F\left(c_{j}\right)} l^{+}(f)$ is the sum of "additions" contained in the files of $c_{j}$, indicating the $d_{i}$ 's total contribution made in the commit $c_{j}$.

(2) $V_{\text {interval }}\left(d_{i}\right)$ : the vector of intervals between two consecutive active behaviors of $d_{i}$, i.e., $V_{\text {interval }}\left(d_{i}\right)=$ $\left\langle\delta_{i 1}, \ldots, \delta_{i\left(\varphi_{i}-1\right)}\right\rangle$ where $\delta_{i j}=t\left(c_{i(j+1)}\right)-t\left(c_{i j}\right)$ measures the time interval between $c_{i j}$ and $c_{i(j+1)}$ both included in $d_{i}$ 's commit sequence $C S_{i}$.

(3) $H_{\text {times }}\left(d_{i}\right), H_{l o c}\left(d_{i}\right)$ : the continuity entropy of active contributing behaviors of a developer $d_{i}$. Entropy has been widely applied in software engineering for various measurement on software entities and software development activities, such as software complexity entropy [5] and software change entropy [6]. Here we borrow this concept to measure how a developer's contributions on source code are distributed over time. The first entropy is "times entropy" measuring the uncertainty in the times distribution of $d_{i}$ 's active behaviors in $N$ timeslots $\left\langle p_{1}, \ldots, p_{N}\right\rangle$, i.e., $H_{\text {times }}\left(d_{i}\right)=-\sum_{k=1}^{N}\left(\frac{\varphi_{i k}}{\varphi_{i}}\right) \log \left(\frac{\varphi_{i k}}{\varphi_{i}}\right)$. The second one is "LoC entropy" which measures the continuity of the LoC in $d_{i}$ 's active behaviors, i.e., $H_{l o c}\left(d_{i}\right)=$ $-\sum_{k=1}^{N}\left(\frac{l_{i k}^{+}}{l_{i}^{+}}\right) \log \left(\frac{l_{i k}^{+}}{l_{i}^{+}}\right)$where $l_{i}^{+}=\sum_{c_{j} \in C S_{i}} l^{+}\left(c_{j}\right)$ is the total LoC that $d_{i}$ has contributed during the lifespan of the project.

For relative timeline, $V\left(r_{k}\right)$ delineates the relative distribution characteristics w.r.t. the releases of a project:

(4) $V\left(r_{k}\right)$ : the vector of the contribution ratios of al1 the developers in one release period $r_{k}$, i.e., $V\left(r_{k}\right)=$ $\left\langle\sigma_{1 k}, \sigma_{2 k}, \ldots, \sigma_{\tau k}\right\rangle$ where $\sigma_{i k}$ is the ratio of $d_{i}$ 's contribution (LoC) relative to the total contributions that all developer$\mathrm{s}$ have made during the period from the release $r_{k-1}$ to $r_{k}$, i.e., $\sigma_{i k}=\frac{l_{i}^{+}\left(r_{k}\right)}{l^{+}\left(r_{k}\right)}$, and $l_{i}^{+}\left(r_{k}\right)=\sum_{c_{j} \in r_{k} \wedge c_{j} \in C S_{i}} l^{+}\left(c_{j}\right)$, $l^{+}\left(r_{k}\right)=\sum_{c_{j} \in r_{k}} l^{+}\left(c_{j}\right), \sum_{k=1}^{\tau} \sigma_{i k}=1$, and $\tau=|T|$ is 


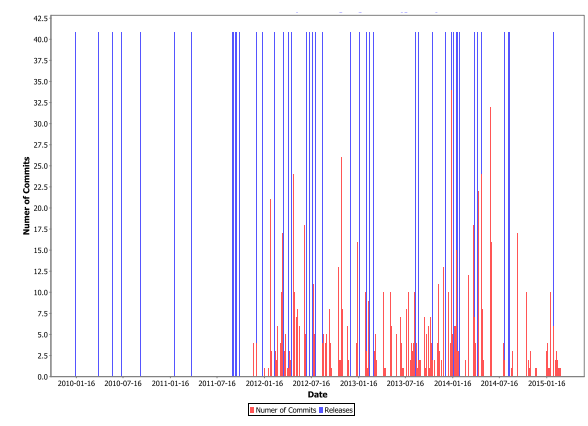

(a) $V_{\text {times }}\left(d_{1}\right)$ of Guava.

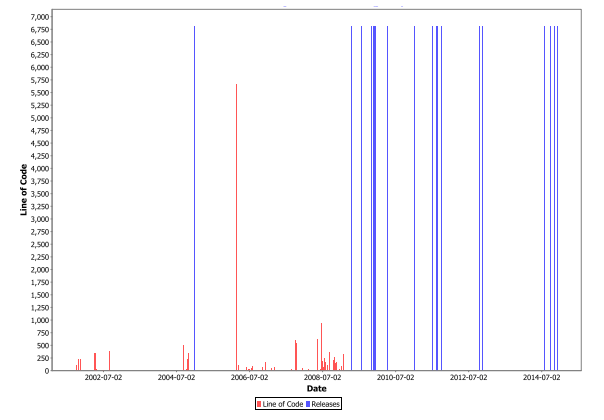

(b) $V_{l o c}\left(d_{2}\right)$ of JUnit.

Figure 1. Barcode visualizing contribution distribution w.r.t. absolute timeline

the total number of developers.

\section{Study Results}

\subsection{Contribution Distribution w.r.t. Absolute Time}

To exhibit the distribution of developers' contribution w.r.t. the absolute timeline, "barcode" is employed to visualize the distribution vectors $V_{\text {times }}\left(d_{i}\right)$ and $V_{l o c}\left(d_{i}\right)$ for each developer $d_{i}$.

Fig. 1 gives the barcode of two developers from JUnit and Guava, respectively. Fig. 1(a) is the times distributions of a Guava developer, and Fig. 1(b) is the LoC distribution of a JUnit developer. The timeline is split into $N$ timeslots (the length of timeslot is 1 day; $N=5,235$ for JUnit and $N=2,003$ for Guava). The red vertical bars are active contributions, and the height of the bars shows the times or LoC of behaviors occurring in the specific timeslot. As a reference, blue bars represent the releases of the project.

Barcode is a straightforward way to visualize the temporal distribution of developers behaviors. Without more precise quantitative metrics, the continuity and the intensity of active behaviors at different times can be intuitively observed from the barcode. For example, $d_{1}$ has intensive and continuous active behaviors from the middle of the project, $d_{2}$ 's active behaviors are split by two long intervals, and $d_{3}$ 's behaviors are

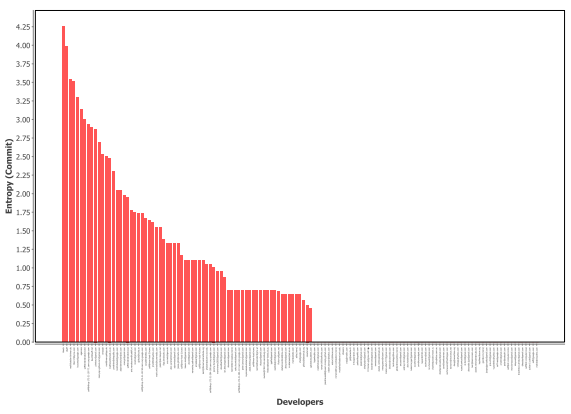

(a) $H_{\text {times }}$ of JUnit.

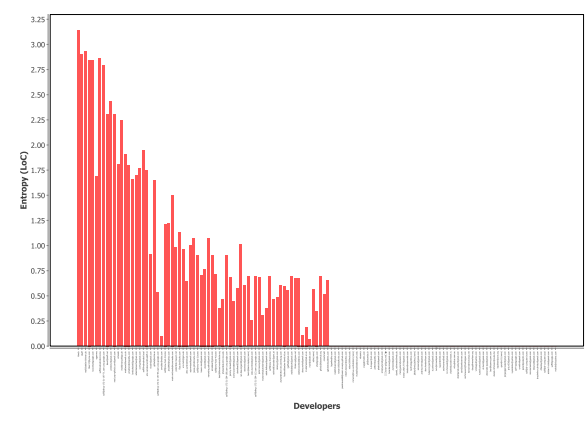

(b) $H_{l o c}$ of JUnit.

\section{Figure 2. Comparison of the continuity entropy among developers}

concentrated in a very short period.

By observation of the barcode for 133 developers in JUnit and 44 in Guava, we find that the shapes of barcode of different developers are quite diversified. Result of Chi-squared test of independence has shown that there are significant difference between the distribution vectors of any pair of developers belonging to the same project. It confirms that the distribution vectors of active behaviors can be used as individualized characteristics of OSS developers.

Fig. 2 demonstrates the continuity entropy $H_{\text {times }}$ and $H_{l o c}$ of developers in JUnit. Developers are sorted by $H_{\text {times }}$ in descending order. A higher entropy indicates that the developer's contributions are distributed over time in a more balanced way. The entropy of some developers in the right equals to 0 because all their active behaviors concentrate in only one timeslot.

From the comparisons between $H_{\text {times }}$ and $H_{l o c}$ for JUnit, and between the ones for Guava, the distributions of $H_{\text {times }}$ and $H_{l o c}$ show almost the same shape. The Spearman's rankorder correlation test shows that the two entropy are highly correlated. For JUnit, the correlation coefficient is 0.863 $(p<0.01)$; for Guava, it is $0.907(p<0.01)$.

Nevertheless, there are still some inconsistencies between them. For example, a developer has higher $H_{\text {times }}$ while his $H_{l o c}$ is comparatively smaller. This implies that, the distribution of LoC in his active behaviors is more unbalanced and 


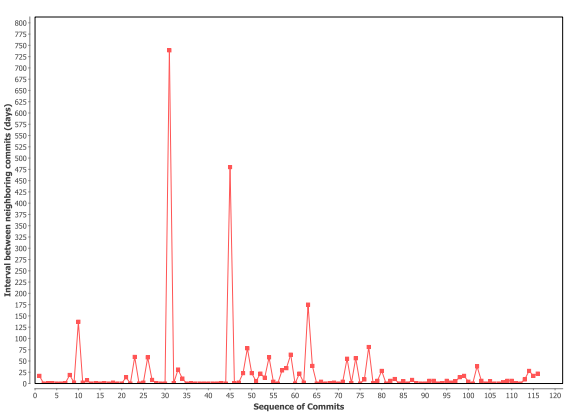

(a) Developer 3 of JUnit.

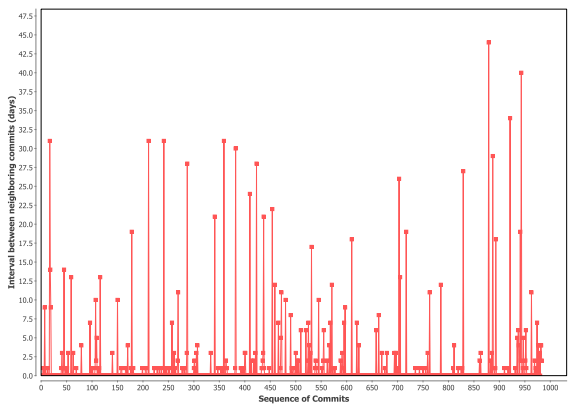

(b) Developer 4 of Guava.

\section{Figure 3. Distribution of intervals between neighboring contributing behaviors}

changes more drastically than the distribution of behavior times. For example, there is a developer in JUnit with almost all his LoC (nearly $45 \mathrm{KLoC}$ ) contributed in the last period of his participation, and the LoC in other periods are very small, thus his $H_{\text {times }}$ is in rank 24 among all developers while his $H_{l o c}$ is in rank 70 only.

We observe from the barcode that, contributions of many developers are distributed in a stage-wise manner, i.e., between two phases of intensive active behaviors, there is usually a long interval. Fig. 3 shows $V_{\text {interval }}$ of two developers in JUnit and Guava, respectively. Although a majority of the intervals are close to $x$-axis (indicating the intervals are short and the contributions are approximately consecutive), there are obviously some outliers that have relatively higher values and represent the long gaps between stages of behaviors. For example, the developer in Fig. 3(a) has two obvious intervals, the first one is one year, and the second is two years. By contract, although Fig. 3(b) seems to have more frequent fluctuations, the amplitudes of these fluctuations are actually smaller than the ones of the first developer (see the different scale of $y$-axis in the two figures).

Summary: (1) Barcode is an intuitive method for visializing temporal distribution of a developer's contributions, and entropy can measure the continuity of contribution distribution; (2) There are temporal locality in the contributions of developers, i.e., most of the intervals between neighboring behaviors are

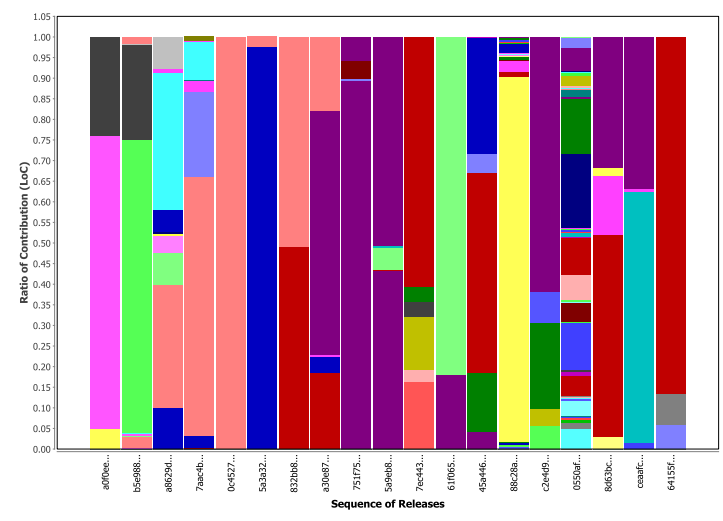

(a) JUnit.

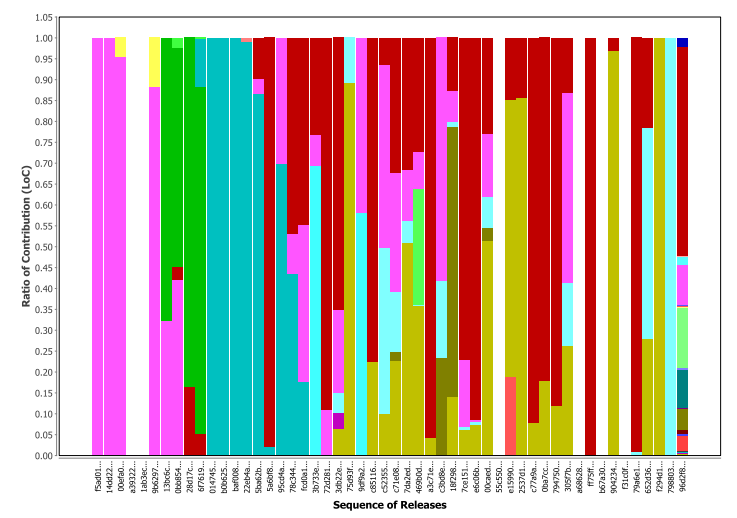

(b) Guava.

\section{Figure 4. Stacked barchart of the relative contri- bution ratio of developers in releases}

short; but there are a small number of long intervals that split the behavior sequence into stages.

\subsection{Distribution of Contributions w.r.t. Releases}

$V\left(r_{k}\right)=\left\langle\sigma_{1 k}, \sigma_{2 k}, \ldots, \sigma_{\tau k}\right\rangle$, vector of the contribution ratios of developers in one release period $r_{k}$, is visualized first. In the form of stacked barchart in Fig. 4 all the releases appear side by side for comparison. The $x$-axis is a set of releases sorted in chronological order, and the $y$-axis is the ratio of contributions. Sub-bars with the same color in different releases represent the same developer.

Some significant facts are observed: (1) There are usually a few developers whose contributions dominate one release. The number of dominant developers is so few that the contributions of other developers involving in the same release eventually become less obvious. (2) A small number of releases possess a large number of developers (e.g., $4^{\text {th }}$ release from the end in JUnit and the last release in Guava). (3) Significant contributions that a developer makes are generally located in one single or multiple consecutive releases.

We use $5 \%$ as the threshold of judging whether a developer 
is a "significant contributor" of a release, i.e., if the ratio of the added LoC of a developer relative to the total added LoC of a release is above $5 \%$, he is considered to be a significant developer of this release. Sensitivity analysis has shown that the value of this threshold ranging from $2 \%$ to $10 \%$ yields similar results.

In JUnit, the range of number of contributors in one release is $[1,59]$, but most of releases have less than 10 developers, above $80 \%$ releases have less than 3 significant contributors, and the contribution ratio of the leading developer is usually far beyond the one of the runner-up. Similar situation can be found in Guava, too. Comparing the two projects we find that the average and median number of contributors of one release in JUnit are both larger than the ones in Guava (average: $9.42>3.30$; median: $5>2$ ), and the average variance of contribution ratios of these contributors in JUnit is smaller than the one of Guava $(0.025<0.04)$. These statistical results show that the participation degree of developers in JUnit is higher than the one in Guava; in other words, developers in JUnit are more active than the ones in Guava.

In the heat chart of Fig. 5, if a developer in $y$-axis is a significant contributor of a release in $x$-axis, the corresponding grid is filled with red color. Developers who are not significant contributors of any releases are not included in the figure. The heart chart further demonstrates the continuity (temporal locality) of the active behaviors of developers relative to the milestones of OSS projects, i.e., for most of developers, their active behaviors dominate one or several releases which are adjacent or close to each other.

To note that, the absolute timespan of different release periods are quite different (see the distance between blue vertical bars in Fig. 1), and the total LoC of different releases are greatly different, too; thus, even if the contributions of a developer dominate a release, it does not indicate that his absolute LoC contribution are large. Once again, what Fig. 4 shows are the ratio of contributions in each release period.

To sum up, the conclusions drawn in this section are: (1) Being the milestones of an OSS project, a majority of releases are dominated by very few developers whose active behaviors constitute the main code changes of the releases. The number of contributors of most releases is usually low, and only very few releases have a large number of contributors whose contributions are relatively balanced. (2) Contributions of a developer tend to be distributed in neighboring or near releases. This demonstrates the temporal locality of a developer's active behaviors relatively to the staged milestones of the project. (3) The distribution of the number of dominating releases of all the developers exhibits approximate power law.

\section{Threats to Validity}

We select DVs in three levels, i.e., the temporal distribution of contributions for the most detailed level; the intensity and

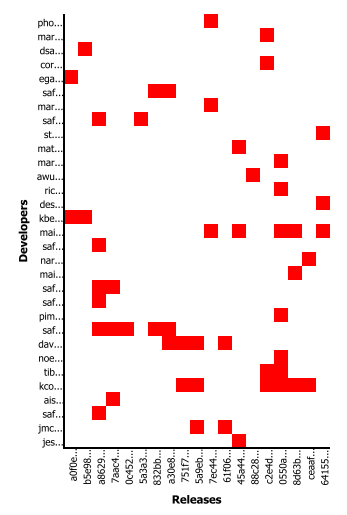

(a) JUnit.

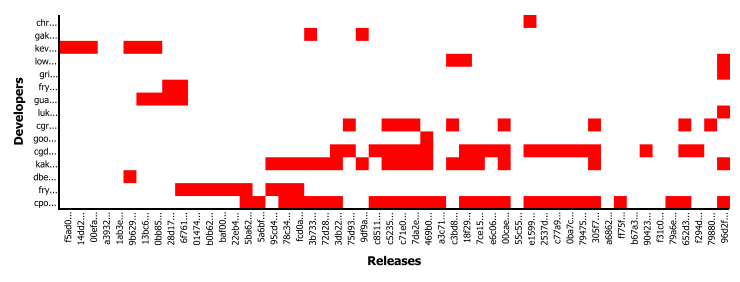

(b) Guava.

\section{Figure 5. Heat chart of significant developers relative to releases}

contribution ratio relative to project releases for the middle level; and the entropy for the global level. These DVs are calculated from the IVs which are derived from the open data of the OSS repositories hosted on Github. Such multi-level DVs ensure the comprehensiveness and understandability of the measurement.

Active behaviors are defined as the contributing activities that developers take on their own initiatives, mainly the committing activities that have explicit code contributions to projects. The exclusion of other types of activities (such as forking a project, commenting, or reporting an issue) from the study would result in some incompleteness of the identified individualized behavioral characteristics. Nevertheless, many existing research adopted similar approaches as ours.

Only the added lines of code in commits are considered but the "deletions" are ignored. However, if a developer removes redundant or buggy code without adding new lines, he still has contributions to the project. It makes the measurement on the continuity of contributions a little biased.

Two OSS projects are selected for the case studies. In an attempt to address the generalizability of our findings, more studies conducted on larger-scale and longer-living OSS projects are necessary.

\section{Related Work}

Code commitment is considered as the dominant behaviors of developers because they take direct effect on the source code 
of OSS projects, e.g., Teyton et al [7] extracted a developer's contribution by delta analysis on source code changes being the result of developer behaviors. Yang et al [8] analyzed the commit activities of developers and found that they are not regularly distributed along with time, thus "barcode" is used to visualize the distribution of commit sequence of each developer. Our study extends their barcode by (1) using the height instead of the width of bars to signify the attributes (times, LoC, and intensity) of behaviors, and (2) putting the barcode into a timeline, so that the temporal distribution of contributions is visualized more precisely.

Siy et al [9] presented a method to summarize work history of developers in terms of the files they have modified over time using the time series segmentation technique, and the segmentation result is used as an individualized feature of developers and some evolution patterns of developer behaviors are found. In our study, we use two types of time segmentation approaches to split the developer behaviors into segments, i.e., absolute and relative timelines.

Lin et al [10] studied the relationship between the distribution of commit behaviors and the releases of OSS projects and identified five distinct zones in the distribution of commit activities across various releases; their conclusion indicates that developer behaviors are not independent but partially influenced by global constraints of projects such as "deadline". In our study, we borrow this idea and use releases as the relative timeline to study the correlation between developers' contributions and project milestones.

Weissgerber et al [11] used a transaction visualization technique to show commit sequences in a project and used fileauthor matrix to visualize evolution history of a file in terms of developers. This approach stands on the file's point of view; by contrast, we use a line of code as basic change unit rather than a large-grained file, thus the result would be more accurate.

\section{Conclusion}

We conduct an case study on the individualized features and common patterns of contributions of OSS developers. Continuity of active contributing behaviors is focused to explore how developer behaviors are distributed over time by a set of finegrained metrics. The conclusions are: (1) Active behaviors of a developer often show temporal locality. Some long intervals split a developer's behaviors into stages, and the more contributions a developer makes, the more evenly his active behaviors are distributed over time, with averagely shorter intervals between neighboring stages of intensive active behaviors. (2) Most of releases of a project are dominated by limited number of developers, respectively, and the total number of contributors in each release is usually low. Active behaviors of a developer tend to be distributed in neighboring or near releases.

The findings of this study would help OSS project coordinators get deep insight in the behavioral characteristics of team members so as to improve their project management practices. Future work will be conducted on this perspective.

\section{Acknowledgment}

Work in this paper is supported by the Natural Science Foundation of China (No. 61272187, 61472106).

\section{References}

[1] J. A. Roberts, I.-H. Hann, and S. A. Slaughter, "Understanding the motivations, participation, and performance of open source software developers: A longitudinal study of the apache projects," Management Science, vol. 52, no. 7, pp. 984-999, 2006.

[2] G. Hertel, S. Niedner, and S. Herrmann, "Motivation of software developers in open source projects: an internetbased survey of contributors to the linux kernel," Research Policy, vol. 32, no. 7, pp. 1159-1177, 2003.

[3] B. J. Dempsey, D. Weiss, P. Jones, and J. Greenberg, "Who is an open source software developer?" Comm. the $A C M$, vol. 45, no. 2, pp. 67-72, 2002.

[4] M. Y. Allaho and W.-C. Lee, "Trends and behavior of developers in open collaborative software projects," in 2014 Int'l Conf. Behavior, Economic and Social Computing. IEEE, 2014, pp. 1-7.

[5] W. Harrison, "An entropy-based measure of software complexity," IEEE Trans. Software Engineering, vol. 18, no. 11, pp. 1025-1029, 1992.

[6] G. Canfora, L. Cerulo, M. Cimitile, and M. Di Penta, "How changes affect software entropy: an empirical study," Empirical Software Engineering, vol. 19, no. 1, pp. 1-38, 2014.

[7] C. Teyton, M. Palyart, J.-R. Falleri, F. Morandat, and X. Blanc, "Automatic extraction of developer expertise," in 18th Int'l Conf. Evaluation and Assessment in Software Engineering. ACM, 2014, p. 8.

[8] W. Yang, B. Shen, and B. Xu, "Mining github: Why commit stops-exploring the relationship between developer's commit pattern and file version evolution," in APSEC'13. IEEE, 2013, pp. 165-169.

[9] H. Siy, P. Chundi, and M. Subramaniam, "Summarizing developer work history using time series segmentation: challenge report," in MSR'08. ACM, 2008, pp. 137-140.

[10] S. Lin, Y. Ma, and J. Chen, "Empirical evidence on developer's commit activity for open-source software projects," in SEKE'13, 2013, pp. 455-460.

[11] P. Weissgerber, M. Pohl, and M. Burch, "Visual data mining in software archives to detect how developers work together," in MSR'07. IEEE, 2007, pp. 9-16. 